\title{
An Automatic Building Reconstruction Method from Airborne LiDAR
}

\section{Data and Maps}

\author{
Li Jing ${ }^{1}$, Xu Siqi ${ }^{23}$, Zhang Wei ${ }^{1}$, Huang Yin ${ }^{23}$, Gao Ping ${ }^{1}$ \\ ${ }^{1}$ Xiamen Great Power GEO Information Technology CO., LTD, Fujian Xiamen 361009 \\ ${ }^{2}$ State Key Laboratory of Information Engineering in Surveying, Mapping and Remote Sensing, \\ Wuhan University, Wuhan 430072, China \\ ${ }^{3}$ Shenzhen R\&D Center of State Key Laboratory of Information Engineering in Surveying, \\ Mapping and Remote Sensing, Shen Zhen, China
}

Keywords: airborne LiDAR; building boundary; building reconstruction; normal segmentation Abstract. To the problem of poor efficiency and low accuracy of building reconstruction method, an automatic building reconstruction method from airborne LiDAR point clouds and building outline from GIS maps is proposed. This article discusses the key steps of building reconstruction such as the buildings extraction, roof segmentation, roof structural line extraction, topology reconstruction and model generation. Finally, to verify the effectiveness and availability of the various steps, a real experiment proved that this method can quickly rebuild a more complex model of city buildings.

\section{Introduction}

Digital city construction has been widely carried out in the medium and large cities at home and abroad, and building model reconstruction is one of the key elements [1]. Airborne LiDAR (Light Detection and Ranging, LiDAR) can quickly obtain the high precision dense lattices of the objects in the Earth's surface, it has become an important method for the three-dimensional digital city model reconstruction [2]. With the increasingly sound and improved of basic surveying and mapping, high-precision and large-scale topographic map data has been completed editing and stored in the database in many places across the country, providing basic information for the national development [3].

In the past 20 years, scholars who use LiDAR data to reconstruct building model has achieved a considerable success [4-5]. Because of the limited resolution of the point cloud data and discrete form of data organization, the outline of the building is often not accurate enough and resulting in a certain deviation from the topographic map data, which is contrary to the consistency requirements of geographic information data [6]. At the same time, building outline is fundamental for the surveying and mapping department, planning department, the 1:500 topographic map of urban areas ,of a wide range and in high precision, has also been a large area coverage [3], which may provide high-precision outline information for three-dimensional building reconstruction using point cloud data.

In summary, the integration of LiDAR data and topographic map can not only take full advantage of existing fundamental mapping results, but also satisfy the real needs of the practical production. Liu Yawen et al [7] provide building outline through topographic map and use a simple parametric model defined in advance to calculate parameters based on the point cloud data, then establish the simple model, and finally generate three-dimensional building model. The key point of this algorithm is to decompose building object into simple models, but in practice, the models will be 
subjected to certain restrictions. Vosselman et al [5] integrate airborne point cloud and topographic data to reconstruct models, in which building outline is directly involved in the topology generation of the model, but the method is not desirable to reconstruct the complex buildings.

In view of the above situation, we propose a building reconstruction method integrated building outline with LiDAR data. By using the existing high-accuracy data of building outline, and extracting building data, segmenting roof surface, detecting structure line, reconstructing topology model through the LiDAR data, this method can quickly generate three-dimensional building models eventually.

\section{Airborne LiDAR building reconstruction integrated outer contour}

The contents of automatically reconstructing building include two aspects namely roof surface segmentation and roof topology reconstruction. Based on building outline footprints from GIS maps, we can extract building point clouds from LiDAR point clouds, and then detect the roof surface point cloud by normal segmentation, to complete the segmentation of the roof surface. On this basis, extracting the roof structure line and obtaining master direction of the building by outline, results are used to constrain structure line. Combined roof structure line with building outline, we can complete the spatial dichotomy merger (Binary Separate Partition, BSP) [8], and then build the two-dimensional topological roof model. Finally, we can match three-dimensional roof patches by the least squares method and get three-dimensional coordinates of angular points in each sheet, then generate three-dimensional building model. Specific method process is shown in Figure 1.

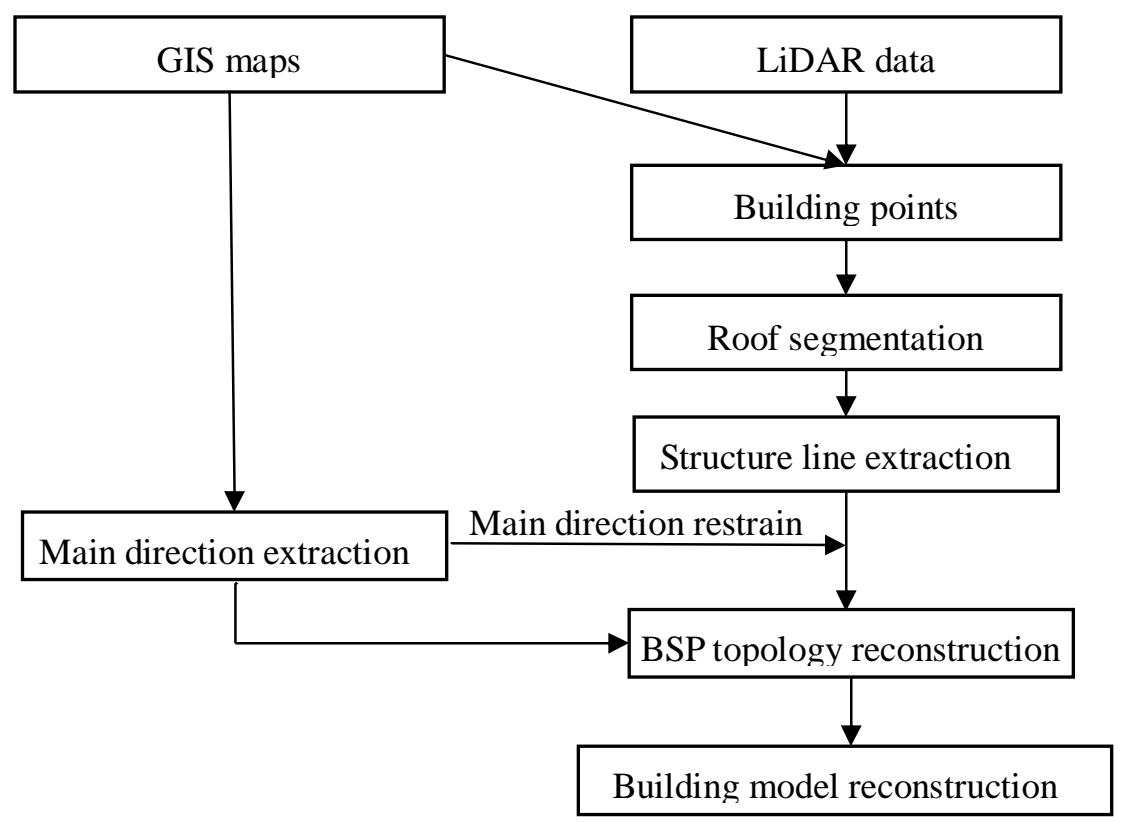

Fig. 1. The workflow of the building reconstruction

\section{Roof Patch Detection}

The detection of roof surface is to split separate roof sets from point cloud sets of the building, the process is divided into following specific steps:1)Using normal segmentation to extract point cloud data of roof surface which is in different directions; 2)Segmentation in heights, namely to separate the point clouds of roof, in same direction at different heights, depending on different spatial distance; 3)Regional clusters, divide the point cloud sets that on the same plane but in the different spatial locations by the methods, and finally get a single roof patch. 
Normal segmentation of point cloud. In space, normal vector of a single point is arbitrary, however point clouds obtain the geometry information of ground surface, normal vector is generally calculated by fitting point sets in a local neighborhood [9]. For point $\mathrm{p}, \mathrm{N}$ points adjacent to it have set $S=\left\{p_{i}, i=1,2, \ldots N\right\}, N \geqslant 3$, normal vector is defined as $n=(a, b, c)$ and $\|\dot{n}\|=1$ (Fig. 1). Considered the distance between normal vector and center point is a negative correlation, to introduce weighting function $\mathrm{w}_{\mathrm{i}}$, so

$$
P(n, d)=\sum_{i=1}^{N} \operatorname{argmin}\left[w_{i} \cdot\left([n, r] \cdot\left[p_{i}, d_{i}\right]^{T}\right)\right]^{2}
$$

where $\mathrm{h}$ is normal vector of plane $\mathrm{P}, \mathrm{d}_{\mathrm{i}}$ is distance between plane $\mathrm{P}$ and original point, weight, $\mathrm{w}_{\mathrm{i}}=\mathrm{w}\left(\left\|\mathrm{p}-\mathrm{p}_{\mathrm{i}}\right\|\right): \mathrm{N}\left(0,2 \mathrm{P}_{\mathrm{dst}}\right), \mathrm{P}_{\mathrm{dst}}$ is the average point distance. After normalizing $\mathrm{P}$ set heart, simplify Eq. 1, we have:

$$
P\left(\begin{array}{l}
r \\
n
\end{array}\right)=\sum_{i=1}^{N} \operatorname{argmin}\left[w_{i} \cdot\left(\stackrel{r}{n} \cdot\left[p_{i}^{\prime}\right]\right)\right]^{2}, \quad\|n \quad\|=1
$$

where $p_{i}^{\prime}=p_{i}-\bar{p}, \bar{p}=\sum_{i=1}^{n} w_{i} p_{i} / \sum_{i=1}^{n} w_{i}$. Hessian matrix is $3 \times 3$ positive semi-definite matrix, which has reliable numerical solution.

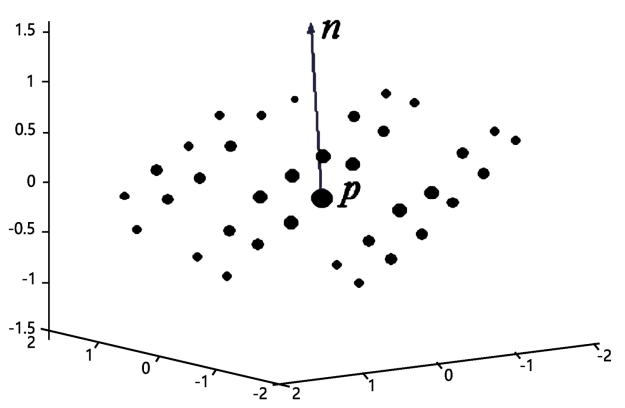

Fig. 2. Weighted normal vector, point size is proportional to weight, $\mathrm{p}$ is center point, $\mathrm{n}$ is normal vector

Weighted normal fitting method has some smoothing effects, but if the smoothing intensity is too high, the method is not conducive to express roof details, and if smoothing intensity is too small, it is vulnerable to be interfered by noise. Since the laser spot data keep changing density in the local area, the neighborhood of a fixed radius size does not match with the actual radius. Therefore, we can use Delaunay triangulation algorithm [10] to build the adjacency of the points (Fig. 3).

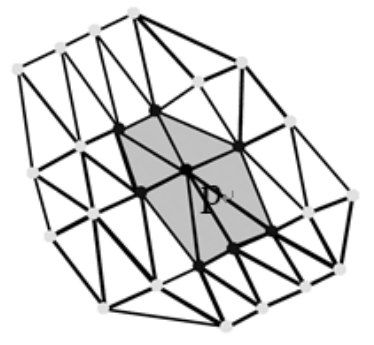

Fig. 4. Point adjacent relation based on Delaunay triangulation, $\mathrm{p}$ is center point, its neighbor is in dark 
Through the method above, normal vector of a single point is complete. To cluster same normal vector, we use Hough transform [11] method to obtain cluster centers, namely for the three components of normal vector $(\mathrm{a}, \mathrm{b}, \mathrm{c})$, to divide at same interval according to the $\lambda_{\mathrm{n}}$ in the parameter space, then transform to numerical space $(i, j, k)$, where:

$$
(\mathrm{i}, \mathrm{j}, \mathrm{k})=\left(\left[\mathrm{a} / \lambda_{\mathrm{n}}\right],\left[\mathrm{b} / \lambda_{\mathrm{n}}\right],\left[\mathrm{c} / \lambda_{\mathrm{n}}\right]\right.
$$

where $a \in[-1,1], b \in[-1,1], c \in[0,1]$. Next, according to voting rules, to transform normal vector of a single point to space (i, j, k) according to Eq. 3. Given poll in (i, j, k) space, to separate point set $\mathrm{S}$ of different normal vector:

$$
\mathrm{Sn}=\{\text { Sni, } \mathrm{i}=1,2, \ldots \mathrm{n}\}
$$

where $\mathrm{Sn}_{\mathrm{i}}$ is the point set which has same normal vector.

Segmentation of distance between point cloud data. After normal segmentation of point cloud data, we get point set in the parallel patch $\operatorname{Sn}_{i}=\left\{\Pi_{i}, i=1,2, \ldots n\right\}, \Pi_{i}$ is point set that has same normal parameters (Fig. 5a). Due to different distance from different patch to origin, to divide distance to the original $d_{i}$ at same interval according to the step-length $\lambda_{n}, d_{j}$ is the number of interval region corresponding to each point, it can be calculated by the Eq. 5 :

$$
\mathrm{d}_{\mathrm{i}}=\left|\left(\begin{array}{l}
\mathbf{u} \\
\left.\mathrm{n}_{\mathrm{i}} \cdot \mathrm{p} / \lambda_{\mathrm{d}}\right)
\end{array}\right)\right|
$$

where $\mathrm{h}$ is normal direction, $\mathrm{p}$ is point coordinate. Same as the normal vector clustering method, we use voting decision ideas to poll in a one-dimensional space and get plane point set $\Pi_{i}$ in different distance to origin (Fig. 6b).

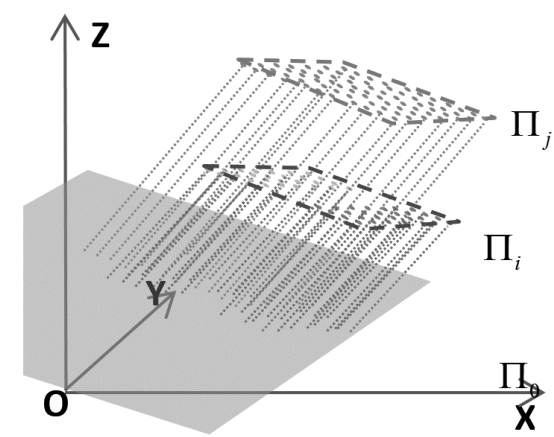

(a) Point set of different origin distance $\Pi_{\mathrm{i}} 、 \Pi_{\mathrm{j}}$

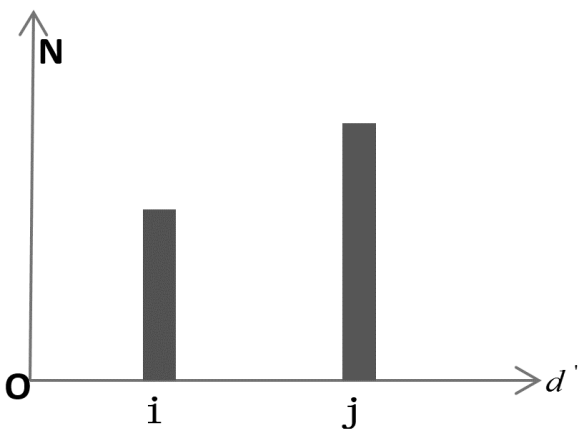

(b)Statistic number of points in the $\mathrm{d}_{\mathrm{i}}$ axis in $\Pi_{\mathrm{i}}, \Pi_{\mathrm{j}}$

Fig. 7. The segmentation of points distance

Segmentation of point cloud region. In the real world, roof surface is a polygonal zone which has boundary. Three-dimensional plane equation is, however, in no boundary planes, which may include several roof surfaces, namely $\Pi=\left\{s_{i}, i=1,2, \ldots n\right\}, s_{i}$ is point set of a single roof surface (Fig. 5), plane $\Pi$ is composed of point sets $s_{1}$ and $s_{2}$. Therefore, to obtain independent roof surface, we need to segment clustering of point cloud region. This paper uses regional growth [12] method to merge 
point cloud within same class and divide point cloud between different class, then separates $\mathrm{s}_{1}$ and $\mathrm{s}_{2}$ in figure 错误! 未找到引用源。.

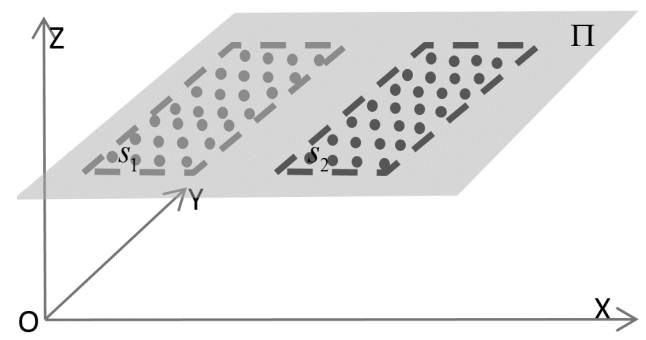

Fig. 8. The plane $\Pi$ includes two point sets $\mathrm{s}_{1}$ and $\mathrm{s}_{2}$

\section{Detection of structure line}

Roof structure line is an important part of the roof topology, including the ridge line and jump line. Extract the main direction of building. The main direction of building is important inner information that can be used to constrain and optimize detected roof structure line. Building outline reflects the information of main direction to some extent. Assume the building outline $B=\left\{l_{i}\right.$, $\mathrm{i}=1,2, \ldots \mathrm{n}\}$ is composed of $\mathrm{N}$ boundary line 1 , we can calculate the angle $\theta$ of each line with the $\mathrm{X}$-axis, namely:

$$
\theta=\left\{\begin{array}{l}
\cos ^{-1}(1(\mathrm{x}) /\|1\|), 1(\mathrm{y})<0 \\
\cos ^{-1}(-1(\mathrm{x}) /\|1\|), 1(\mathrm{y})<0
\end{array}\right.
$$

where $\theta$ is the angle between 1 and $X$-axis, $1(x)$ is the $x$ component of $1,1(y)$ is the $y$ component of 1.We digitize $\theta$, and then do peak statistics to get peak value that meets the threshold condition $\mathrm{T}_{\theta}$, and then use the sets of normal vector that within these peaks to calculate the main direction of building.

Extract roof ridge line. Roof ridge line is the intersection line of adjacent roof pieces, it is important boundary information to distinguish two different roof facets. For inclined adjacent roof surface $r_{i}$ and $r_{j}$, straight line $\mathrm{l}_{\mathrm{ij}}$ is intersection line (Fig. 6a).

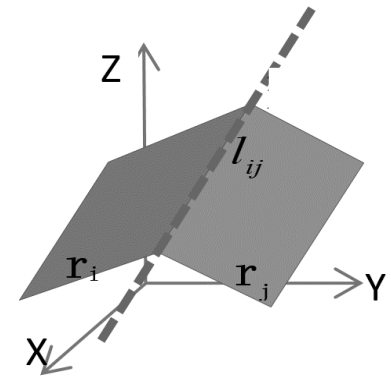

(a) Intersection line $\mathrm{l}_{\mathrm{ij}}$ of roof surface $\mathrm{r}_{\mathrm{i}}$ and $\mathrm{r}_{\mathrm{j}}$

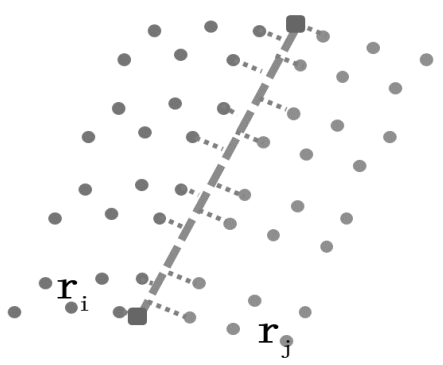

(b) Connection point of $r_{i}$ and $r_{j}$ to determine roof ridge line

Fig. 6. Ridge edge line extraction

After fitting the space linear equation of roof ridge line, we determine the end point of roof ridge line by the point projection in the intersecting lines ridge line, the point is near the sheet, and then detect roof ridge line (Fig. 6b). 
Extract jump line. Jump line is the common edge where elevation of roof sheets has step change in space. The key to extract jump line is to find the corresponding elevation changing points. There is a certain tilt angle during the acquisition process of laser scanning data, the roof surface which is far from the sensor and at lower elevation is easy to be sheltered by the adjacent higher facets. Therefore, the roof edge point at higher elevation is defined as a jump point.

Assume point $\mathrm{p}$ and $\left\{\mathrm{p}_{\mathrm{i}}, \mathrm{i}=1,2, \ldots \mathrm{M}\right\}$ is adjacent, if point $\mathrm{p}$ meets Eq. 7 , we define $\mathrm{p}$ as jump point.

$$
\left\{\begin{array}{l}
\left|\min \left(\mathrm{p}_{\mathrm{z}}-\mathrm{p}_{\mathrm{iz}}\right)\right|>\left|\max \left(\mathrm{p}_{\mathrm{z}}-\mathrm{p}_{\mathrm{iz}}\right)\right| \\
\max \left(\mathrm{p}_{\mathrm{z}}-\mathrm{p}_{\mathrm{iz}}\right)-\min \left(\mathrm{p}_{\mathrm{z}}-\mathrm{p}_{\mathrm{iz}}\right)>\mathrm{T}_{\mathrm{j}}
\end{array}\right.
$$

where point $p_{z}$ is the $z$ coordinate of point $p$, point $p_{i z}$ is the $z$ coordinate of point $p_{i}, T_{j}$ is the given threshold (Fig. 7).

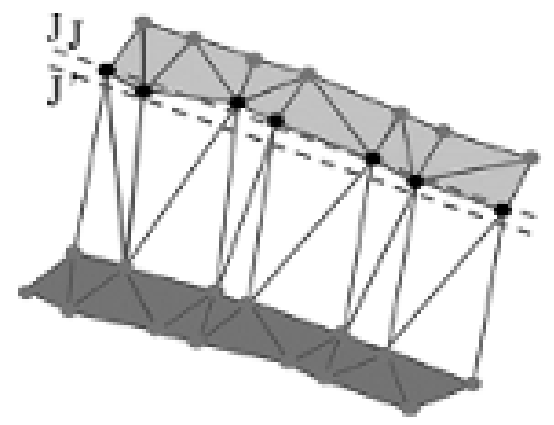

Fig. 9. Jump points are in dark color, $\mathrm{J}$ is fitted jump edge by least square method and $\mathrm{J}$ ' is the final jump edge

Due to the limited ground resolution of point cloud and laser spot can not necessarily express the building edge information, the segment results fitted by the least squares method do not accurately describe the real edge. Thus, there will be a more accurate result when jump line appropriately translates outwards to a certain distance according to the outermost point, as J, J' in figure 错误! 未 找到引用源。.

\section{Reconstruction of BSP topology}

Jump line and ridge line offers roof structure features, building outline provides building edge feature, but these features are not connected together to form closed area and an effective geometric topology mechanism. The method of BSP is to do the bivariate subdivision with space [13], which can be used to rebuild geometric topology structure by roof structure line and building outline [8], specific process can be divided into split and merge steps.

Split. BSP split, namely a space is divided into two sub-spaces, each sub-space will be subdivided into two subspaces, such iterative process will continue until the condition is not satisfied the division. In this paper, building outline is used as an initial division of space, roof structure line as a condition to divide space, whether has structure line within the space as condition that whether to continue dividing process.

In the division process, structure line is used to divide polygon, and point clouds in space is divided into different sub-spaces, the entire process is shown in figure 8. This article defines the structure that composed by polygon and internal point clouds as BSP block, the category which the largest number of point clouds has in the BSP block as BSP block properties (Fig. 8c). 


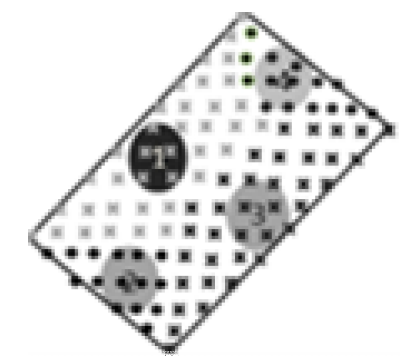

(a) Segmentation result and corresponding number

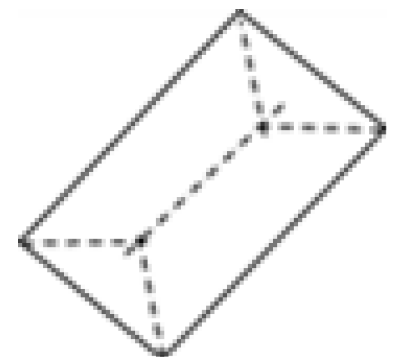

(b)The outline and structure line

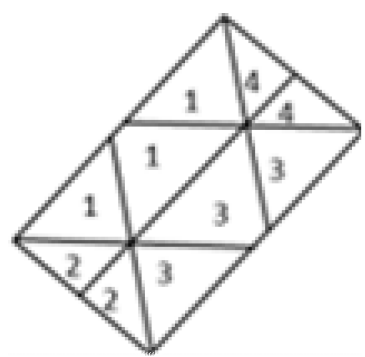

(c)Division results and BSP block properties

Fig. 10. The partition and merging process of building roof

Merger. It means to merge BSP blocks that belong to same piece of roof surface. After split step, the roof is divided into many BSP blocks, since BSP block is labeled by property values, merger process only merges the blocks that have same value to a single BSP block (Fig. 11). Property values of BSP block are affected by the BSP block size. BSP block, which has larger area, more points inside, and the effects of noise and boundary point is relatively small to the whole BSP type, has relatively stable properties. The BSP, which is smaller blocks and has less internal points, is susceptible to noise when it determines attribute.

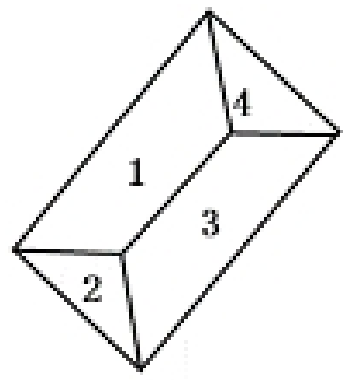

Fig. 12 Results of merging the BSP block with some attribute

Model generation. After BSP merger, there is a group of BSP blocks which contains roof outline shape and point set of roof surface. Inside each BSP, points of most types are used to fit parameters of roof surface by the least squares method [14] and solve $Z$ coordinates of polygon corner point projected on the plane, thus roof surface can be calculated into three-dimensional space.

After calculating three-dimensional structure of roof surface, you need to generate the building wall, which can be classified into two kinds of external and internal walls. External wall can directly use ground projection of building contour, ground level of current building can be obtained by $\mathrm{Z}$ value of point in a certain neighborhood around the building. Internal walls appear on the roof step line, and it is the plane projection from large elevation plane to small one (Fig. 13).

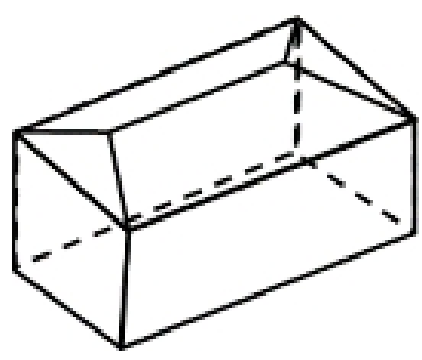

Fig. 14 The model generation by least square roof fitting 


\section{Experiment and analysis}

In this paper, the experimental data was selected in Tianjin, got by the company Optech's ALTM Gemini LiDAR Mapping System. There are different kinds of buildings and complex structure in the experimental data, and point clouds in this area has been preprocessed, such as classification. In this area, the average density of point cloud is 1.75 (points $/ \mathrm{m}^{2}$ ), 1819527 points totally, including 407280 points of buildings, 556 points of building outline. Partial experiment results are shown in figure 11. The overall effect is shown in figure 12.

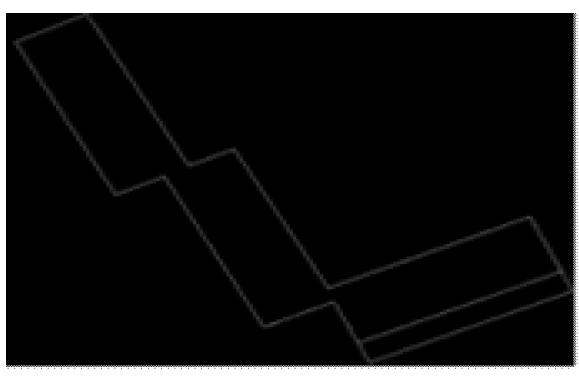

(a)The outline of the building

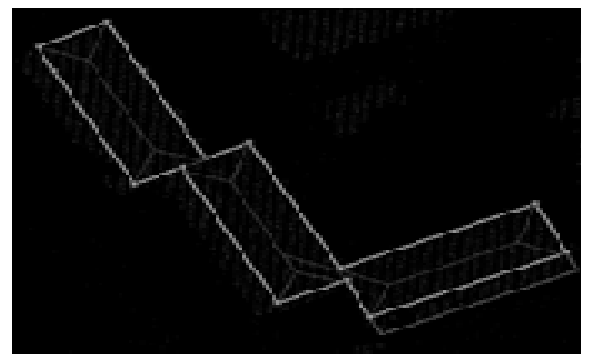

(c) Reconstruction result of BSP topology

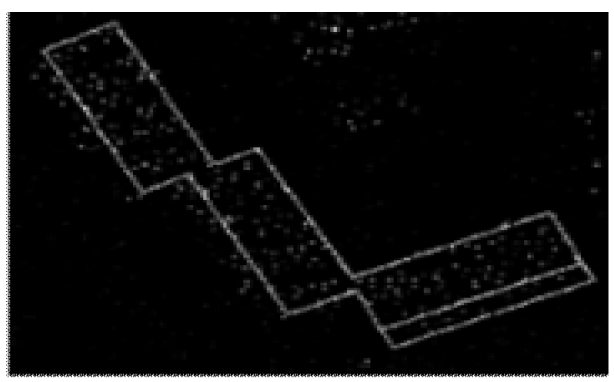

(b)The outline and point cloud of building

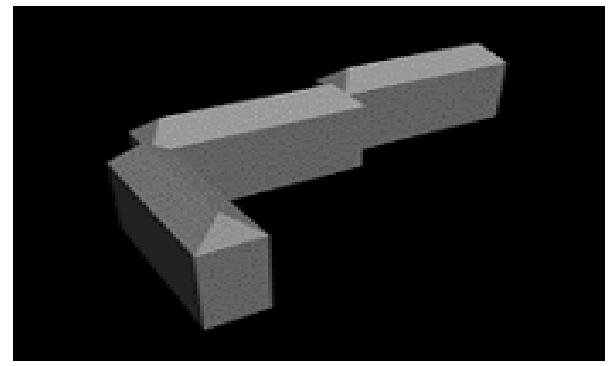

(d) The result of three-dimensional reconstruction

Fig. 15. The results of each reconstruction step

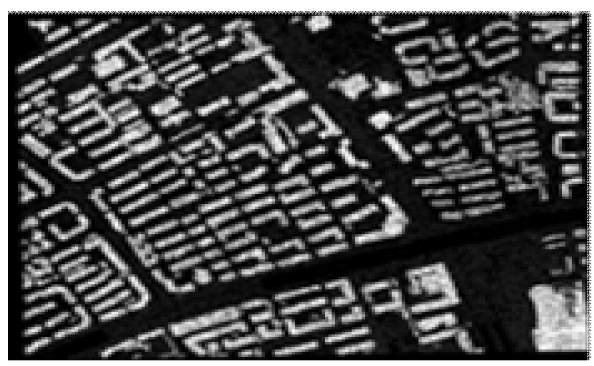

(a) Point cloud of experimental area

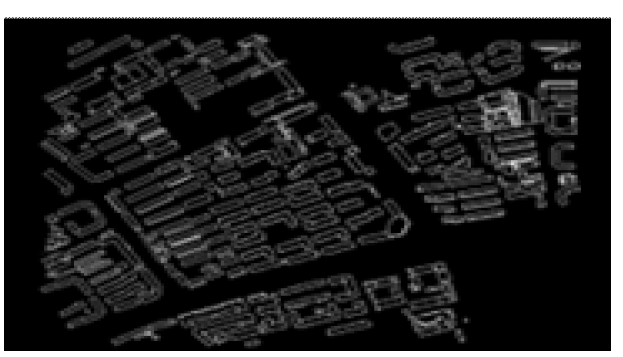

(b) The building outline corresponding to experimental area

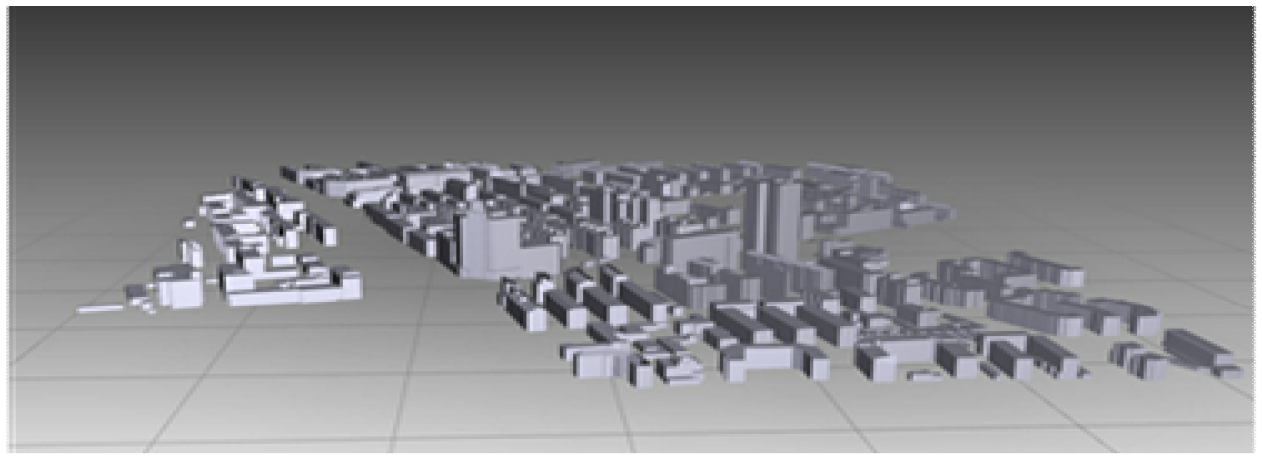

(c) three-dimensional models of the building

Fig. 16 Result of the building reconstruction 
Results by this method are shown in figure 11(d) and 12 (c). Method realization is integrated in the self-developed software, which is embedded the Micro Station V8i system by the form of plug-ins. Experiment on Windows XP system which contains intel-i3-2100 dual core processor and 4GB memory has generated 532 models, taken 192 seconds, 446 correct reconstructions, 86 not completely rebuilt.

\section{Conclusion}

This paper proposes an automatically modeling way which merges building outline with airborne laser point cloud, the method detects model patch, extracts structure line, reconstructs BSP topology, and generates building model in the end. It's able to complete better building reconstruction that is not so complex and has obvious advantages on time efficiency and strong practicability. This paper has tested the generating method of building outline and airborne laser point cloud, experiment shows that the method is feasible, it can greatly improve modeling efficiency. The method has some shortcomings and areas for improvement:

(1) For the complex roof structure which contains more patches and small area, we have to extract more line structure and roof structure stability is poor, easily causes BSP over-segmentation;

(2) For the detailed roof structure, such as dormer windows, elevator and so on, it's hard to rebuild these based on data-driven. We will consider the approach of combining image and model-driven in our later work, adding the criterion of symmetry and regularity constraint to rebuild detailed structure, it is need to do more research.

\section{Acknowledgments}

This research work is supported by the National Natural Science Foundation of China (41571437) and Dedicated Foundation of Developing Strategic Emerging Industries of Shenzhen program (No. JCYJ20140421102206026、JCYJ20120618163005494)

\section{References}

[1] Li D, Shao Z, Yang X: The Theory and practice from Digital City to Smart City [J]. Geospatial Information. Vol. 9 (2011), p. 1-5.

[2] Feng M: Discussion on Three-dimensional Modeling Based on LiDAR Data and Aerial Images [J]. Bulletin of Surveying and Mapping. Vol. 12 (2011), p. 12-14.

[3] Song X: An Effective Way for Innovation of Basic Large-scale Map [J]. Bulletin of Surveying and Mapping. Vol. 11 (2004), p. 25-26.

[4] Sun Y, Zhang X, Luo G: Improved Active Contour Model for Building Roof Boundary Extraction from LiDAR Point Cloud. Acta Geodaetica et Cartographica Sinica. Vol. 6 (2014), p. 620-626.

[5] Vosselman G: Building Reconstruction Using Planar Faces In Very High Density Height Data [J]. Iaprs. Vol. 32(1999), p. 87-92.

[6] Hu Y, Zhang Z, Lin H: Feasibility Research of Large Scale Digital Topographic Mapping Using Airborne LiDAR. Bulletin of Surveying and Mapping. Vol. 05 (2015), p. 87-90.

[7] Liu Y, Song S: An Approach on Building Reconstruction from Images Data Clouds and Vector Maps [J]. Geomatics and Information Science of Wuhan University. Vol. 34(2009), p.894-897.

[8] Sohn G, Huang X, Tao V: Using a Binary Space Partitioning Tree for Reconstruction Polyhedral 
Building Models from Airborne Lidar Data [J]. Photogrammetric Engineering \& Remote Sensing. Vol. 74(2008), p.1425-1440.

[9] Li B, Cheng Z, Dang G, Jin S: Survey on normal estimation for 3D point clouds [J]. Computer Engineering and Applications. Vol. 46 (2010), p.1-7.

[10] Wu X, Wang S, Xiao C: A new study of Delaunay triangulation creation [J]. Acta Geodaetica et Cartographica Sinica. Vol. 28 (1999), p.28-35.

[11] Hough V, Paul C: Method and means for recognizing complex patterns. US3069654 [P]. 1962.

[12] Hu H, Cui H, Dai X: Segmentation of scattered point data based on region growing method [J]. Journal of Computer Applications. Vol. 29(2009), p.2716-2718.

[13] Tao Z, Cheng C, Pan Z, Shi J: Generation and Applications of a Multi-Resolution BSP Tree [J]. Journal of Software. Vol. 01 (2011), p.117-125.

[14] Yuan Q, Lou L, Chen W: Applying Weighted Total Least-Squares to the Plane Point Cloud Fitting of Terrestrial Laser Scanning [J]. Bulletin of Surveying and Mapping. Vol. 3(2011), p.1-3. 